\title{
Organ-Specific Effects of Oxygen and Carbogen Gas Inhalation on Tissue Longitudinal Relaxation Times
}

\author{
James P.B. O’Connor, ${ }^{1,2}$ Alan Jackson, ${ }^{1}$ Giovanni A. Buonaccorsi, ${ }^{1}$ David L. Buckley, ${ }^{1}$ \\ Caleb Roberts, ${ }^{1}$ Yvonne Watson, ${ }^{1}$ Sue Cheung, ${ }^{1}$ Deirdre M. McGrath, ${ }^{1}$ \\ Josephine H. Naish, ${ }^{1}$ Chris J. Rose, ${ }^{1}$ Paul M. Dark, ${ }^{3}$ Gordon C. Jayson, ${ }^{2}$ and \\ Geoff J.M. Parker ${ }^{1}$
}

\begin{abstract}
Molecular oxygen has been previously shown to shorten longitudinal relaxation time $\left(T_{1}\right)$ in the spleen and renal cortex, but not in the liver or fat. In this study, the magnitude and temporal evolution of this effect were investigated. Medical air, oxygen, and carbogen $\left(95 \%\right.$ oxygen $\left./ 5 \% \mathrm{CO}_{2}\right)$ were administered sequentially in 16 healthy volunteers. $T_{1}$ maps were acquired using spoiled gradient echo sequences (TR $=3.5 \mathrm{~ms}$, TE $=0.9 \mathrm{~ms}$, $\alpha=2^{\circ} / 8^{\circ} / 17^{\circ}$ ) with six acquisitions on air, 12 on oxygen, 12 on carbogen, and six to 12 back on air. Mean $T_{1}$ values and change in relaxation rate were compared between each phase of gas inhalation in the liver, spleen, skeletal muscle, renal cortex, and fat by one-way analysis of variance. Oxygen-induced $T_{1}$-shortening occurred in the liver in fasted subjects $(P<0.001)$ but not in non-fasted subjects $(P=0.244)$. $T_{1}$-shortening in spleen and renal cortex (both $P<0.001$ ) were greater than previously reported. Carbogen induced conflicting responses in different organs, suggesting a complex relationship with organ vasculature. Shortening of tissue $T_{1}$ by oxygen is more pronounced and more complex than previously recognized. The effect may be useful as a biomarker of arterial flow and oxygen delivery to vascular beds. Magn Reson Med 58:490-496, 2007. () 2007 Wiley-Liss, Inc.
\end{abstract}

Key words: biomarker; carbogen; longitudinal relaxation time; magnetic resonance imaging; oxygen; physiology

Molecular oxygen $\left(\mathrm{O}_{2}\right)$ has two unpaired electrons and is paramagnetic, with a magnetic moment of 2.8 Bohr magnetons. For this reason, $\mathrm{O}_{2}$ shortens tissue longitudinal relaxation time ( $T_{1}$ time) and increases signal intensity on $T_{1}$-weighted sequences (1). Thus, while under standard conditions dissolved plasma oxygen plays a negligible role in oxygen transport in human physiology (2), the $T_{1}$-shortening effect provides a mechanism for monitoring oxygen delivery to tissues, by detecting signal modulation in plasma and tissue fluids that contain dissolved oxygen.

${ }^{1}$ Imaging Science and Biomedical Engineering, University of Manchester, Manchester, UK.

${ }^{2}$ Cancer Research UK Department of Medical Oncology, Christie Hospital, Manchester, UK.

${ }^{3}$ Department of Intensive Care Medicine, Salford Royal National Health Service (NHS) Trust, Hope Hospital, Stott Lane, Salford, UK.

Grant sponsor: Cancer Research UK Clinical Research Training Fellowship Grant; Grant number: C19221/A6086; Cancer Research UK Programme Grant; Grant number: C237/A6295; Grant sponsors: AstraZeneca; GlaxoSmithKline.

*Correspondence to: Dr. James O'Connor, Cancer Research UK/Royal College of Radiologists Clinical Research Training Fellow, Imaging Science and Biomedical Engineering, Stopford Building, University of Manchester, Oxford Road, Manchester, M13 9PT, United Kingdom. E-mail: james.o'connor@manchester.ac.uk

Received 7 November 2006; revised 11 May 2007; accepted 7 June 2007.

DOI 10.1002/mrm.21357

Published online in Wiley InterScience (www.interscience.wiley.com).

(c) 2007 Wiley-Liss, Inc.
The effect is distinct from the blood oxygen level dependent mechanism of contrast, which measures the relative change in deoxygenated hemoglobin (paramagnetic) to oxyhemoglobin (diamagnetic) on $T_{2}^{*}$-weighted imaging.

Normal subjects breathing room air (approximately 21\% $\mathrm{O}_{2}$ ) have a systemic arterial plasma oxygen partial pressure $\left(\mathrm{pO}_{2}\right)$ of $98 \mathrm{~mm} \mathrm{Hg}$ and systemic venous plasma $\mathrm{pO}_{2}$ of $39 \mathrm{~mm} \mathrm{Hg}$. Approximately $1.5 \%$ of oxygen carried by the arterial blood is freely dissolved in the plasma. Inhalation of $100 \%$ oxygen has a minor effect on the systemic venous $\mathrm{pO}_{2}$, which rises slightly to $48 \mathrm{~mm} \mathrm{Hg}$ in healthy subjects. However the $\mathrm{pO}_{2}$ of arterial blood plasma closely matches that of alveolar $\mathrm{pO}_{2}$, which increases from $98 \mathrm{~mm} \mathrm{Hg}$ to approximately $600 \mathrm{~mm} \mathrm{Hg}$ when breathing 100\% oxygen (3). Since dissolved oxygen concentration is directly proportional to the $\mathrm{pO}_{2}$ in plasma, the concentration of dissolved oxygen increases from $0.3 \mathrm{ml}$ per $100 \mathrm{ml}$ to $1.8 \mathrm{ml}$ per $100 \mathrm{ml}$ on $100 \%$ oxygen. Despite this six-fold increase in oxygen carriage, dissolved plasma oxygen still accounts for a relatively small fraction of total oxygen transported in the arterial blood at supranormal $\mathrm{pO}_{2}$ levels. This reflects the relatively large volume of oxygen carried bound to hemoglobin molecules, which are already around 98\% saturated while breathing room air (4).

Several investigators have evaluated oxygen-induced shortening of $T_{1}$ relaxation time on magnetic resonance imaging (MRI) as a form of contrast enhancement in studies of pulmonary physiology (5-7) and pathology (8). One study has demonstrated a linear relationship between both plasma and whole blood $\mathrm{pO}_{2}$ and reduction in $T_{1}$ relaxation times at $1.5 \mathrm{~T}$ (9). The effect of $T_{1}$-shortening has also been reported in cardiac muscle, spleen, and renal cortex, while the effect has not been demonstrated in other organs including the liver and subcutaneous fat $(10,11)$. Furthermore, the mechanism of contrast has been explored in an animal tumor model under hyperbaric conditions and has shown promise as a noninvasive quantitative method of detecting change in $\mathrm{pO}_{2}$ in both normal tissues and tumors (12).

The primary objective of this study was to investigate the $T_{1}$-shortening effect of dissolved molecular oxygen in upper abdominal organs of healthy volunteers using a temporally-resolved 3D volume acquisition. We hypothesized that $T_{1}$-shortening could be measured in a robust manner relatively free from motion corruption and that specific measures of $T_{1}$-shortening might be identified for development as biomarkers of the microvasculature that reflect arterial blood flow and tissue oxygen delivery. Since literature reports suggest that $100 \%$ oxygen may 
cause vasoconstriction in peripheral organs (13) leading to reduction in tissue blood flow (14), we compared the $T_{1}$ reduction on $100 \%$ oxygen with that achieved with carbogen $\left(95 \% \mathrm{O}_{2} / 5 \%\right.$ carbon dioxide $\left.\left(\mathrm{CO}_{2}\right)\right)$, which is believed to counteract oxygen-induced vasoconstriction (15).

\section{MATERIALS AND METHODS}

\section{Subjects}

Approval for the study was granted from the local Research Ethics Committee (Ref: 05/Q1410/124). Smokers, individuals with significant heart or lung disease, and those with contraindication to MRI were excluded (8). A total of 16 healthy normal volunteers (six female and 10 male) who had given written informed consent were included in the study. The mean age was 30.4 years (range 23-44 years).

\section{Circuit Design}

Gases were administered via a non rebreathing circuit. In the first seven volunteers a Hudson mask (Henleys Medical, Welwyn Garden City, Hertfordshire, UK) was used. In the last nine volunteers a continuous positive airway pressure (CPAP) mask with head straps (Vital Signs, Totowa, NJ, USA) was used with a non-rebreathing T-piece circuit without a CPAP valve, with the aim of increasing the inspired oxygen fraction. The procedure and circuit were explained to the volunteer prior to commencement and care was made to ensure a tight seal. During the experiment, all volunteers breathed medical air $\left(21 \% \mathrm{O}_{2}\right)$ followed by $100 \%$ oxygen and then carbogen in the same order and according to the same time schedule. All gases were delivered at 15 liters $/ \mathrm{min}$. Pulse rate was recorded.

\section{Data Acquisition}

All experiments took place on a Philips Intera system (Philips Medical Systems, Best, Netherlands) at 1.5 Tesla, at the University of Manchester Wellcome Trust Clinical Research Facility. The whole body transmit/receive coil was selected for transmission and reception. Initial scout images were followed by a $T_{2}$-weighted single-shot turbo spin-echo image $\left(\mathrm{TR}=606.5 \mathrm{~ms}, \mathrm{TE}=80 \mathrm{~ms}, \alpha=90^{\circ}\right.$, field of view $(\mathrm{FOV})=375 \mathrm{~mm}$, matrix size $=256 \times 256$, slice thickness $=4 \mathrm{~mm}$ ). During this period volunteers breathed medical air in order to acclimatize to the circuit.

Series of 3D $T_{1}$-weighted fast field echo images were acquired over a 9.9-cm volume slab through the upper abdomen to allow estimation of tissue $T_{1}$ relaxation times $\left(\mathrm{TR}=3.5 \mathrm{~ms}, \mathrm{TE}=0.9 \mathrm{~ms}, \alpha=2^{\circ} / 8^{\circ} / 17^{\circ}, 3\right.$ averages, bandwidth $=500 \mathrm{~Hz}, \mathrm{FOV}=384 \mathrm{~mm}$, matrix size $=128 \times$ 128, slice thickness $=3 \mathrm{~mm}$ ). The FOV was selected to include liver, spleen, kidney, paraspinal muscle, and subcutaneous fat. Measurements were acquired during gentle breathing without breathholding. No fat suppression was applied. Total acquisition time for each $T_{1}$ measurement was 76.8 s. Six baseline measurements were collected while breathing medical air, followed by 12 on $100 \%$ oxygen, and then 12 on carbogen. In eight volunteers, six to 12 further measurements were recorded inhaling med-

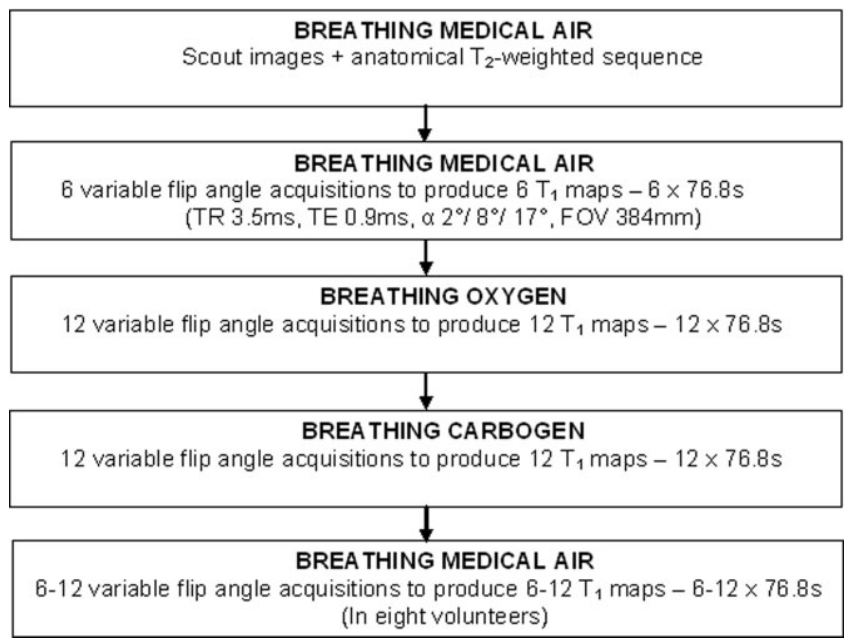

FIG. 1. Summary of 3D fast field echo $T_{1}$-weighted protocol.

ical air after the carbogen phase. The schedule is summarized in Fig. 1.

\section{Image Analysis}

Data were processed in three stages using a voxel-by-voxel fitting process with in-house software. $T_{1}$ maps at each time point were generated using the variable flip angle method $(16,17)$. Representative volumes of interest (VOI) were drawn for each organ by one observer (J.O'C). Mean values (calculated from the six baseline $T_{1}$ maps) and change from baseline for all time points were calculated and compared with those quoted in the literature $(10,11,18,19)$.

The change in oxygen concentration at each time point (denoted $\Delta\left[\mathrm{O}_{2}\right](t)$ ) can be expressed in relation to the longitudinal relaxivity constant for oxygen $\left(r_{1}\right)$, the $T_{1}$ value at each time point $(t)$ and the baseline $T_{1 \text { (baseline), according to }}$ the equation

$$
\Delta\left[O_{2}\right](t)=\frac{\left(\frac{1}{T_{1}(t)}\right)-\left(\frac{1}{T_{1(\text { baseline })}}\right)}{r_{1}} .
$$

Hence, the change in longitudinal relaxation rate $\left(1 / T_{1}(t)\right.$ - $\left.1 / T_{1 \text { (baseline) }}\right)$ - hereafter denoted $\Delta R_{1}$, where $R_{1}$ is the longitudinal relaxation rate-was calculated in each organ VOI for every volunteer at all time points. Therefore, $\Delta R_{1}$ was proportional to the change in tissue oxygen concentration at each time point. Group mean $\Delta R_{1}$ were plotted for each VOI to evaluate the wash-in of oxygen and carbogen and the washout of carbogen. Measurements of $\Delta R_{1}$ for each subject VOI were grouped into the six baseline air measurements, the last 10 oxygen, and the last 10 carbogen measurements to evaluate the difference in tissue $T_{1}$ between the three phases of gas inhalation. The first two oxygen and carbogen time points were excluded from this analysis since the first 2-3 minutes were considered to represent a transition phase of gas wash-in (14). Significance was tested by a one-way analysis of variance with a post hoc Bonferroni correction. For the purpose of analysis 
for the liver VOI, volunteers were split according to fasting status, into those who had fasted for $4 \mathrm{~h}$ or more (fasted group) and those who had eaten or drunk nonclear fluids over the same time (non-fasted group). The baseline $T_{1}$ values for these subgroups were compared using a twosided independent samples $t$-test.

\section{RESULTS}

Pulse rates were significantly reduced on breathing oxygen compared with medical air (64/min vs. 69.5/min; $P=$ 0.001 , paired $t$-test) but not with carbogen inhalation. VOI were drawn in five organs in each volunteer where possible: spleen, liver, renal cortex, paraspinal muscle, and subcutaneous fat. Data were excluded from two volunteers-one due to circuit failure and one where motion between acquisitions made VOI comparison unreliable. A total of 13 volunteers experienced dyspnea breathing carbogen, which resulted in curtailing the examination in one volunteer. No other adverse events were reported.

Mean $T_{1}$ values on air were consistent with those reported in the literature. Representative $T_{1}$ maps for two volunteers are shown in Fig. 2 . The changes in $R_{1}$ from the two circuits were not significantly different in any of the organ VOI-all subsequent results are presented together for all 16 volunteers. No significant difference was present between the baseline liver $T_{1}$ for the fasted (594 $\pm 35 \mathrm{~ms}$, $N=7)$ and non-fasted (570 $\pm 60 \mathrm{~ms}, N=5)$ groups $(P=$ 0.314). Mean $T_{1}$ and $\Delta R_{1}$ for each phase of gas inhalation are shown in Table 1 . Mean group wash-in $\Delta R_{1}$ are presented in Fig. 3.

Both oxygen and carbogen induced highly significant increases in $R_{1}$ in the spleen, liver (in fasted subjects), renal cortex, and paraspinal muscle (all $P<0.001$ ). There was no significant $\Delta R_{1}$ following gas inhalation measured in the liver in volunteers who had eaten within the last $4 \mathrm{~h}$. No difference in $\Delta R_{1}$ between medical air-oxygen and medical air-carbogen was observable in muscle $(P=1.0)$ or renal cortex $(P=0.471)$. Increase in $R_{1}$ in the spleen was significantly greater with oxygen inhalation than with carbogen $(P<0.001)$, whereas increase in $R_{1}$ in the liver in fasted subjects was significantly greater during the carbogen phase of breathing $(P<0.001)$. Significant $R_{1}$ increase was also seen in the signal in subcutaneous fat for oxygen $(P=0.011)$ and carbogen $(P=0.002)$. However, magnitudes of standard error on the group measurement, which may include both true measurement error and organ $T_{1}$ heterogeneity, were considerably greater in fat than in other tissues (Fig. 3f).

The effect of returning to breathe medical air after the air-oxygen-carbogen protocol (washout phase) was examined in the spleen and liver (fasted group). Change in $R_{1}$ with carbogen-medical air in the spleen was highly significant $(P<0.001)$. The mean $R_{1}$ measured while breathing medical air at the beginning and end of the experiment showed no statistical difference $(P=0.407)$, suggesting that the effect of hyperoxic gases is real but short lived in the spleen (Fig. 4a). In contrast, $R_{1}$ in the liver (fasted group) remained unchanged during the second set of acquisitions on medical air from that of carbogen $(P=1.0)$ and was highly significantly different from the original baseline medical air measurements $(P<0.001)$ (Fig. 4b).

\section{DISCUSSION}

The main objective of this study was to evaluate the $T_{1}$ shortening effect of dissolved molecular oxygen in plasma and tissue fluid in the abdominal organs of healthy volunteers. The secondary objective was to compare the $T_{1}$ shortening effect of oxygen with that obtained with carbogen. To our knowledge the effect of carbogen on tissue $T_{1}$ has not been previously investigated.

\section{Oxygen-Induced $T_{1}$-Shortening in Abdominal Organs}

Tadamura et al. (10) studied oxygen-induced $T_{1}$ changes using inversion recovery sequences. Images were acquired breathing room air and then at $220 \mathrm{~s}$ after breathing $100 \%$ oxygen, through a non-rebreathing circuit at a rate of 10 liters/min. Reductions in measured $T_{1}$ in the spleen $(4.2 \%)$, myocardium $(3 \%)$, and arterial blood $(11.3 \%)$ were described. No changes were seen in the liver, dorsal skeletal

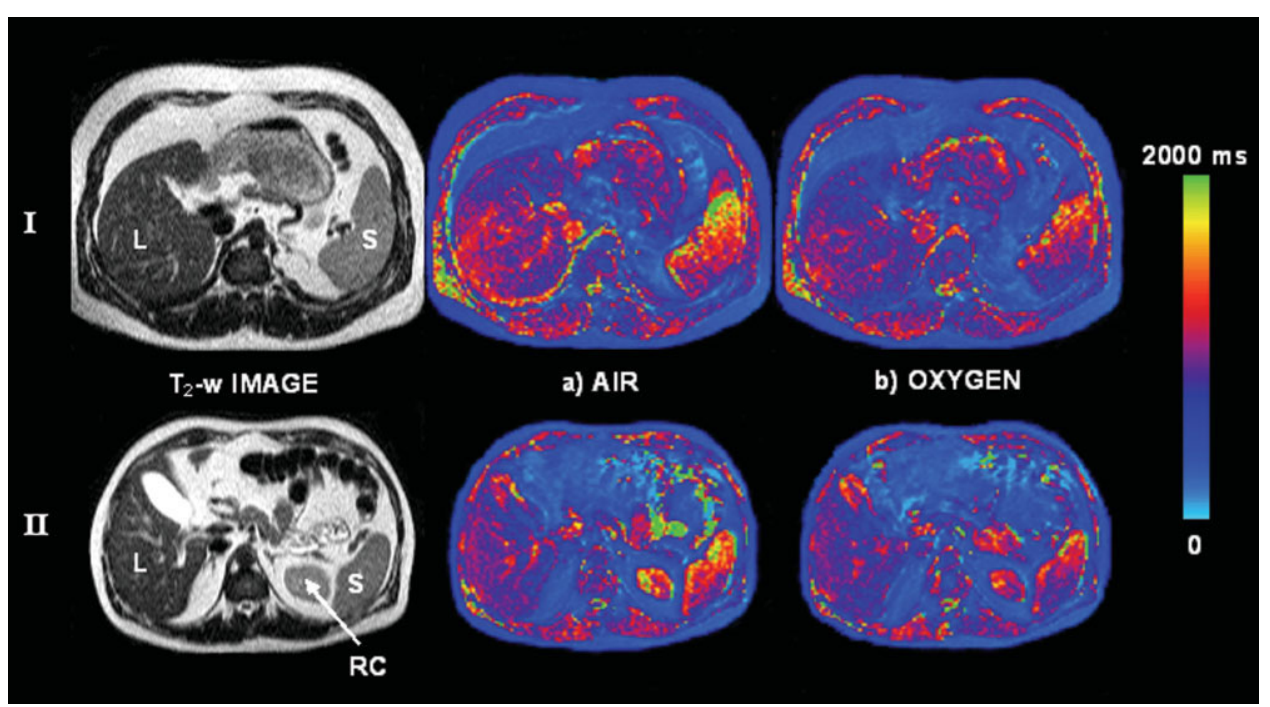

FIG. 2. $T_{2}$-weighted spin-echo axial image with calculated $T_{1}$ maps on (a) air and (b) 100\% oxygen through the upper abdomen in two subjects. A representative slice from a $3 D$ acquisition is shown in each case. In the first subject (I) who had fasted, the $T_{1}$ in the spleen and liver are clearly shortened following inhalation of oxygen. $T_{1}$-shortening can also be observed in the spleen and renal cortex of the second subject (II) who had eaten recently; note that the liver does not appear to change its $T_{1}$ in this individual. Small $T_{1}$ changes in the paraspinal muscle and subcutaneous fat occurred in both volunteers but are not easily identified in these maps. 
Table 1

Mean \pm Standard Error $T_{1}$ Values of Air (6 time points), Oxygen (time points 3-12), and Carbogen (time points 3-12) Inhalation for Each Organ Volume of Interest (VOI) With $N$ Subjects*

\begin{tabular}{|c|c|c|c|c|c|c|}
\hline \multirow[b]{2}{*}{ Organ } & \multirow[b]{2}{*}{$N$} & \multicolumn{3}{|c|}{ Mean $T_{1}$ values $(\mathrm{ms})$} & \multicolumn{2}{|c|}{ Mean $\Delta R_{1}\left(\mathrm{~s}^{-1}\right)$} \\
\hline & & Air $T_{1}$ & $\begin{array}{l}\text { Oxygen } T_{1} \\
\text { (reduction) }\end{array}$ & $\begin{array}{c}\text { Carbogen } T_{1} \\
\text { (reduction) }\end{array}$ & $\begin{array}{l}\text { Oxygen } \\
\text { ( } P \text { value })\end{array}$ & $\begin{array}{l}\text { Carbogen } \\
(P \text { value })\end{array}$ \\
\hline Spleen & 14 & $926 \pm 26$ & $795 \pm 24(14.1 \%)$ & $836 \pm 25(10.0 \%)$ & $0.188(P<0.001)$ & $0.127(P<0.001)$ \\
\hline Liver (not fasted) & 5 & $570 \pm 27$ & $566 \pm 27(0.8 \%)$ & $561 \pm 21(1.6 \%)$ & $0.016(P=0.244)$ & $0.019(P=0.124)$ \\
\hline Liver (fasted) & 7 & $599 \pm 13$ & $580 \pm 11(3.1 \%)$ & $562 \pm 15(6.2 \%)$ & $0.053(P<0.001)$ & $0.113(P<0.001)$ \\
\hline Paraspinal muscle & 11 & $736 \pm 16$ & $709 \pm 16(3.7 \%)$ & $709 \pm 19(3.7 \%)$ & $0.055(P<0.001)$ & $0.057(P<0.001)$ \\
\hline Renal cortex & 5 & $945 \pm 15$ & $883 \pm 9(6.6 \%)$ & $873 \pm 22(7.6 \%)$ & $0.076(P<0.001)$ & $0.092(P<0.001)$ \\
\hline Subcutaneous fat & 12 & $236 \pm 8$ & $232 \pm 8(1.8 \%)$ & $231 \pm 8(2.1 \%)$ & $0.077(P=0.011)$ & $0.091(P=0.002)$ \\
\hline
\end{tabular}

*Mean percentage reduction of $T_{1}$ from air-to-oxygen and air-to-carbogen is given in parentheses. Mean $\Delta R_{1}$ is calculated for each organ on oxygen and carbogen and $P$ values shown are for significant difference from air-oxygen and air-carbogen.

muscle of the back, subcutaneous fat, or bone marrow. A second group reported significant change in arterial blood $T_{1}(17.9 \%)$, but saw no significant change in skeletal muscle (gastrocnemius and soleus) (20). Jones et al. (11) used $T_{1}$-weighted multishot turbo spin-echo sequences to evaluate response to $100 \%$ oxygen inhalation at 15 liters $/ \mathrm{min}$, demonstrating reduction in the $T_{1}$ of both renal cortex $(6 \%)$ and spleen $(8.8 \%)$, but not in the renal medulla or liver. All of the above studies were performed at 1.5 Tesla.

We studied the $T_{1}$-shortening effect of $100 \%$ oxygen in the spleen, renal cortex, liver, paraspinal muscle, and subcutaneous fat. Reductions in spleen $T_{1}$ were reproducible across the group of volunteers and greater than previously described in the literature $(10,11)$. Changes in the renal cortex following oxygen inhalation were of similar magnitude to those reported elsewhere (11). However, difficulty in accurate VOI definition, due to organ motion, limited the number of volunteers with a reliable dataset. Calculation of the change in $T_{1}$ relaxation time can be misleading since the magnitude of change is heavily dependent on the baseline $T_{1}$ value for each organ. Therefore, we converted reduction in $T_{1}$ to $\Delta R_{1}$ since this quantity both normalizes the data and provides a measure of change that is, in theory, proportional to the change in tissue oxygenation, as expressed in Eq. [1].

Previous studies have not shown oxygen-induced $T_{1}$ shortening in the liver $(10,11)$. In this study, we demonstrated a highly significant increase in $R_{1}$ in the liver when volunteers fasted for over $4 \mathrm{~h}$. This effect was absent in those volunteers who had recently eaten. No difference in the baseline $T_{1}$ values were demonstrated between these two groups. Approximately 20 to $30 \%$ of liver blood supply is from the hepatic artery and the remainder from the hepatic portal vein (HPV) although the relative supply of the two vessels is reciprocal and changes according to fasting status (21). Previous studies have attributed the lack of observed change in liver $T_{1}$ relaxation time to its predominantly portal blood supply, since HPV blood has already passed through the splanchnic capillary bed before entering the liver, whereas the $\mathrm{pO}_{2}$ of hepatic arterial
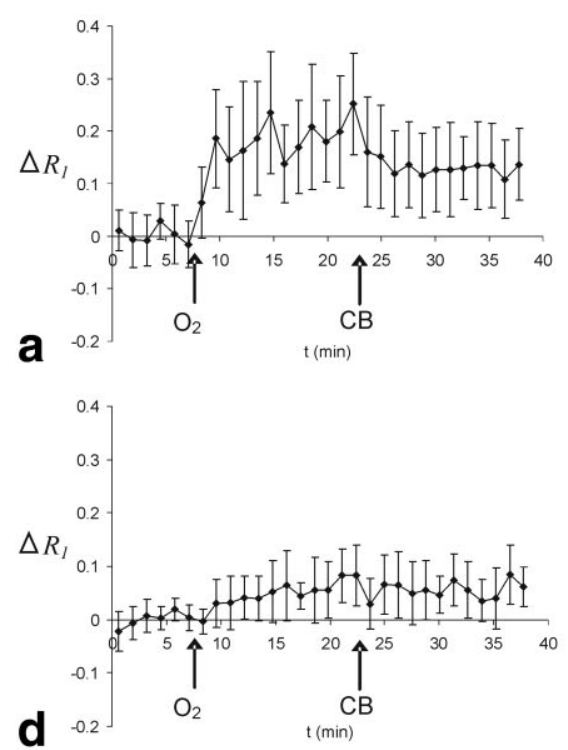
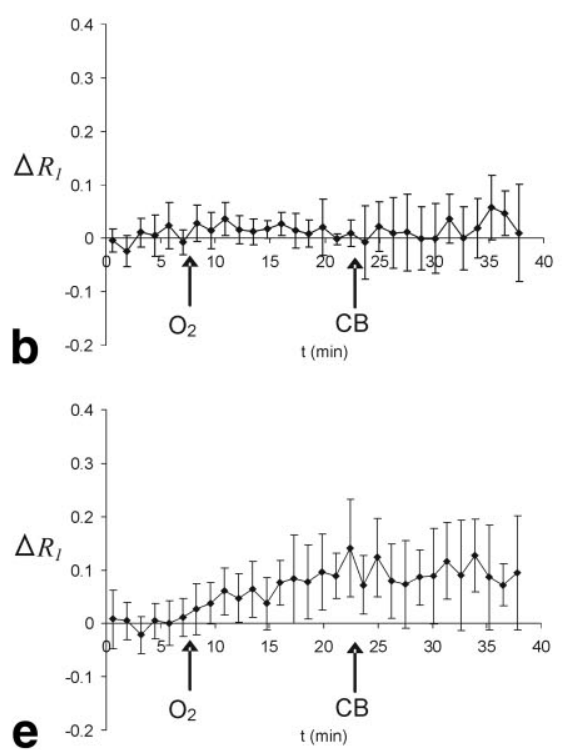
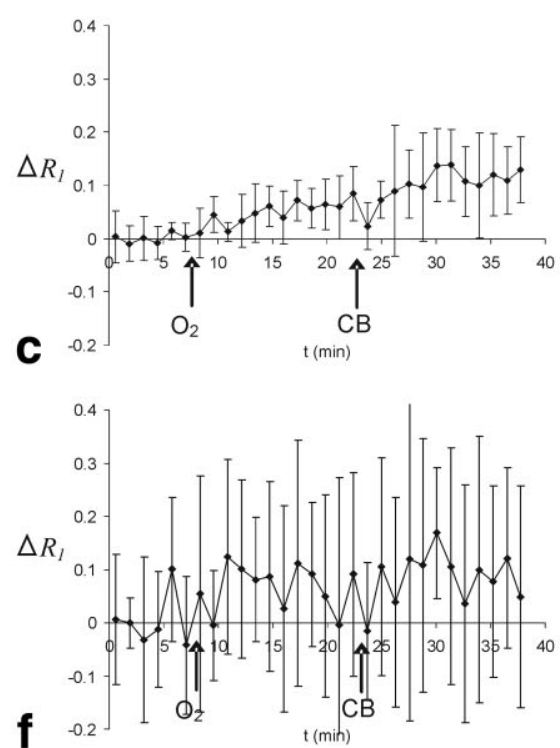

FIG. 3. Mean $\Delta R_{1}$ for each organ volume of interest (error bars are SD across individuals) for (a) spleen, (b) liver (not fasted), (c) liver (fasted), (d) paraspinal muscle, (e) renal cortex, and (f) subcutaneous fat. Equivalent scales have been used to emphasize differences in magnitudes of change and the confidence in measured values for each organ. The start of oxygen $\left(\mathrm{O}_{2}\right)$ and carbogen $(\mathrm{CB})$ inhalation are marked by arrows. 

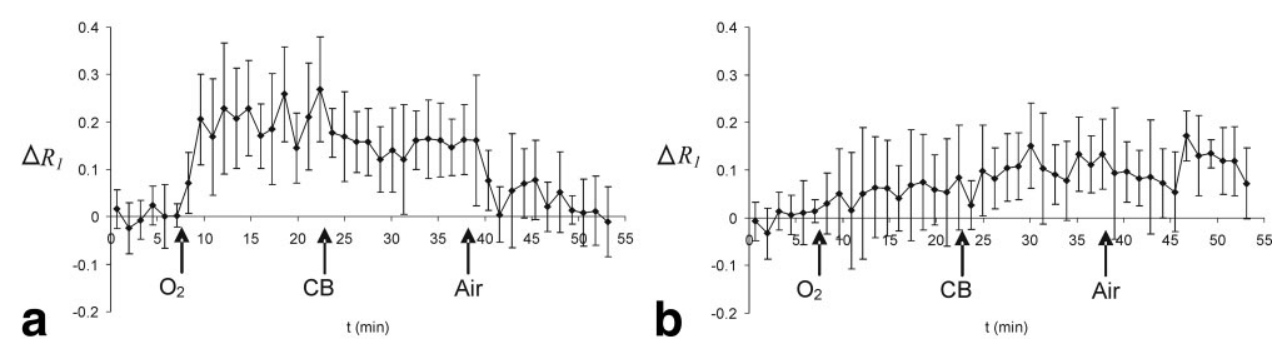

FIG. 4. Mean change in (a) $R_{1}$ in spleen $(N=7)$ and (b) liver (fasted group, $\left.N=4\right)$. Arrows indicate the beginning of breathing oxygen $\left(\mathrm{O}_{2}\right)$, carbogen (CB) and the second phase of breathing medical air (Air). In the case of the spleen, $T_{1}$ values return to that of the original baseline acquisitions once oxygen and carbogen are replaced by medical air. This is not seen within the liver, where the $\Delta R_{1}$ persists for at least 15 min after administration of the hyperoxic gases.

blood may be increased while breathing $100 \%$ oxygen (10). We interpret our results as representing a difference in the relative arterial fraction of hepatic blood flow between the fasted and non-fasted groups; significant $T_{1^{-}}$ shortening can be observed with a larger arterial fraction of hepatic blood flow (e.g., when fasted).

We also measured significant increase in $R_{1}$ in skeletal muscle and in subcutaneous fat. Changes in rotator cuff muscle $T_{1}$ have been previously demonstrated by our group using an inversion-recovery technique (22) in contrast with other studies, but no previous study has described oxygen-induced $T_{1}$-shortening in fat. The changes observed in the fat were considerably noisier than in other organs, which may reflect the lower baseline $T_{1}$ relaxation time of fat compared with the other organs. They were, however, highly significant (Fig. 3f). The discrepancies between our results and other studies may reflect one or more of variation in circuit design (maximum achievable fraction of inspired oxygen), measurement timing, data analysis, and physiological factors.

\section{Physiological Mechanisms Underlying Gas-Induced $T_{1}$-Shortening}

The physiological changes underlying the changes in $T_{1}$ observed at supraphysiological levels of blood plasma oxygenation are complex. Elevation in the amount of dissolved oxygen present in capillaries and tissue fluid (via diffusion) are major contributing factors to the shortening of $T_{1}$ observed within each voxel (11). Current understanding of oxygen delivery to tissues suggests that inhalation of $100 \%$ oxygen elevates arterial plasma $\mathrm{pO}_{2}$ at the level of the proximal arterioles, where a significant reduction in plasma $\mathrm{pO}_{2}$ begins to occur (23). Both the rate and magnitude of oxygen extraction is believed to be organ-specific and has a complex relationship to blood flow and metabolic demand (24). These observations suggest that the $\Delta R_{1}$ observed in this experiment may not only reflect the paramagnetic effect of dissolved molecular oxygen, but may be dependent on tissue metabolism, arterial (and arteriolar) flow, and blood volume within each organ, since a sufficiently elevated amount of dissolved molecular oxygen must be delivered to cause measurable $T_{1}$-shortening.

Decreased blood volume, mediated by oxygen-induced vasoconstriction, may lessen the ratio of blood to tissue within a given voxel. Since arterial blood has a longitudinal relaxation time of around $1.25 \mathrm{~s}(20,25)$, the observed $T_{1}$ within a voxel will reduce to a variable extent, depending on the proportion of blood and tissue within each voxel and the relative change in blood volume. However, it is unlikely that decreased flow with $100 \%$ oxygen would produce changes of sufficient magnitude to account for all of the observed signal change. Previous work from Tadamura et al. (10) suggested that the $T_{1}$-shortening observed is predominantly from the presence of excess oxygen acting as a relaxation agent, rather than by altering organ perfusion. Furthermore, blood flow and volume have been reported to increase in some organs following hyperoxemia (26).

An approximate calculation of maximum $\Delta R_{1}$ from decreased blood volume can be made using a simplistic model of free water exchange between compartments. For an organ (such as muscle or fat) with less than $5 \%$ blood volume (27) a reduction by $20 \%$ would lead to a $\Delta R_{1}$ of around $0.006 \mathrm{~s}^{-1}$. More vascular tissues with a higher percentage blood volume may demonstrate a larger $\Delta R_{1}$, but these are still considerably smaller effects than is observed in all tissues in our study (Table 1).

In this study a 3D acquisition was used. Since all blood flowing in has been sufficiently saturated in the outer parts of the volume, then modest changes in blood flow are unlikely to alter tissue $T_{1}$ in regions of interest drawn in the center of the volume. Therefore, pure (i.e., blood volume-independent) flow changes are unlikely to contribute toward the change in $\Delta R_{1}$ described in our study. Nevertheless, the alteration in blood flow resulting from gas inhalation could be measured using alternative techniques to provide a complete characterization of gas-induced physiological changes.

\section{Difference of Carbogen Gas and 100\% Oxygen}

We compared the measured $T_{1}$-shortening effects of carbogen and $100 \%$ oxygen. Several literature reports describe vasoconstriction in peripheral tissues within minutes following inhalation of $100 \%$ oxygen $(13,14)$. Vasoconstriction may result in reduction of local blood flow by approximately $10-20 \%$ within minutes (28), although this effect is not seen in all human tissues to the same magnitude. Vasoconstriction leads to a transient elevation in blood pressure before homeostatic mechanisms reduce heart rate and therefore cardiac output, hence returning blood pressure to resting levels.

Carbogen and other hyperoxic gases that contain a small percentage of $\mathrm{CO}_{2}$ have been widely used in studies at- 
tempting augment plasma $\mathrm{pO}_{2}$ and tissue oxygen delivery, particularly in the field of radiotherapy (29). The ability of carbogen to counteract oxygen-induced vasoconstriction has been most widely cited as a justification for this strategy, although shift of the oxygen-hemoglobin disassociation curve to the right (the Bohr effect) and increasing cardiac output have also been credited as possible mechanisms (30-32). Optimal carbon dioxide content of the gas mixture remains controversial (33) — the original rationale for using $5 \%$ was empirical rather than experimentallybased (15) — carbogen is difficult to tolerate (32) and in practice circuit limitations produce actual inspired gas concentrations of around $3 \% \mathrm{CO}_{2}, 70 \%$ oxygen, and $27 \%$ nitrogen (34). Furthermore, the effect of carbogen on both normal and pathological tissues is complex and incompletely understood. While carbogen is generally purported to increase perfusion, there is evidence that carbogen decreases flow in healthy tissues (35) and in tumor vasculature (36).

In our experiment, the $T_{1}$-shortening observed in the spleen while breathing $100 \%$ oxygen-measured by increase in $R_{1}$-was significantly reduced once volunteers switched to breathing carbogen (Fig. 3a). Assuming that the dissolved plasma oxygen was $95 \%$ of that obtained during $100 \%$ oxygen inhalation, this differential must represent either reduced blood flow to the spleen (relative vasoconstriction), reduced arterial blood volume, or altered oxygen extraction. This is inconsistent with the hypothesis that carbogen counteracts oxygen-induced vasoconstriction through a direct endothelial response to $\mathrm{CO}_{2}$, raising the possibility of an indirect $\mathrm{CO}_{2}$ effect, for example altered sympathetic nervous system activity.

In contrast to the results obtained in the spleen, $T_{1^{-}}$ shortening in the liver (fasted subjects) was greater with carbogen inhalation than with $100 \%$ oxygen (Fig. 3c). This could either represent the continuing effect of oxygen inhalation (i.e., change in $R_{1}$ was yet to plateau and was not a direct $\mathrm{CO}_{2}$ effect), or suggest a $\mathrm{CO}_{2}$-mediated change in hepatic blood flow or blood volume, either through direct effects on the vasculature, or indirectly. No significant difference in the magnitude of $T_{1}$-shortening on $100 \%$ oxygen or carbogen was detected in skeletal muscle, renal cortex, or in fat (Fig. 3d-f). Finally, the washout effect of carbogen appears to vary between the liver and spleen (Fig. 4).

Thus, the effect of carbogen inhalation does not appear to produce equivalent responses in all organs and may reflect complex homeostatic mechanisms, as well as the direct effect of carbon dioxide on the tissue endothelium. For example, the liver differs from other tissues in having a dual blood supply and in having fenestrated capillaries rather than capillaries lined with a collar of smooth muscle that changes its caliber in response to oxygen and $\mathrm{CO}_{2}$ levels (2). In addition, while $\mathrm{CO}_{2}$ may counteract oxygeninduced vasoconstriction in some organs, elevated $\mathrm{CO}_{2}$ levels can decrease regional blood flow to the kidney (37), having an opposing effect. Furthermore, elevated $\mathrm{CO}_{2}$ levels may induce vasodilatation in skeletal muscle, by acting upon nitric oxide receptors in capillary smooth muscle (38). Therefore, several competing factors may influence any flow and blood volume contributions to $\Delta R_{1}$ within each organ.

\section{Study Design and Limitations}

Compromise between spatial resolution, temporal resolution, and signal-to-noise ratio must be made in an exploratory imaging study of this kind. The spatial resolution used in this experiment precluded voxel-by-voxel comparison; therefore, mean values of $T_{1}$ and $\Delta R_{1}$ were calculated. Each time point represented data collected over a period of $76.8 \mathrm{~s}$ in order to obtain a signal-to-noise ratio sufficient to detect change in $R_{1}$. Six acquisitions were repeated to accurately establish a baseline $T_{1}$ value for each organ VOI. It was decided that the first two time points following switching a gas would be considered to represent the time to equilibrium, since this period (just over $150 \mathrm{~s}$ ) is consistent with experiments of oxygen physiology (14) and the reported time for oxygen and carbogen plateau in other experiments (39-41). The two circuits used did not produce significantly different results.

The question of whether the continued reduction of $T_{1}$ in the liver (fasted subjects) was due to prolonged oxygen wash-in or $\mathrm{CO}_{2}$ response could have been answered by modifying the protocol to intersperse the oxygen and carbogen phases with a second period breathing medical air. However, total acquisition time was one hour, complying with ethical considerations of subject tolerability. Also, oxygen and carbogen wash-in times for organs are unknown. For these reasons the order of medical air-oxygencarbogen was selected for all subjects.

Our observations raise several interesting points regarding future studies of oxygen induced $T_{1}$-shortening with MRI. Oxygen inhalation was well tolerated by all subjects. The technique can measure an approximation of regional oxygen concentration that is relatively simple to calculate and enables continuous in vivo measurements to be made. While previous studies have suggested small changes in $T_{1}$ following oxygen inhalation, the magnitudes of $\Delta R_{1}$ during different phases of gas inhalation were sufficient to be detected, suggesting that $\Delta R_{1}$ may be a usable measure of tissue oxygen delivery for use in future studies of both normal physiology and pathology. Further studies are required to evaluate the relationship between change in arterial plasma flow and blood volume-as evidenced by the fasting-specific changes in the liver-and change in $R_{1}$. Furthermore, the MRI measure $\Delta R_{1}$ following oxygen inhalation requires validation against histological measures of tissue oxygenation.

\section{CONCLUSION}

We have demonstrated a change in tissue longitudinal relaxation time with both oxygen and carbogen in a variety of normal tissues. The technique is robust, well tolerated, and allows organ-specific $T_{1}$-shortening to be calculated in individual organs. Larger alterations in $T_{1}$ were seen than described in previous studies and changes have been described in organs previously considered not to exhibit $T_{1}$-shortening. It has also highlighted differences in tissue physiology between different organs, the explanations of which are complex and require further elucidation.

\section{ACKNOWLEDGMENTS}

J.P.B.O.C. is funded by a Cancer Research UK Clinical Research Training Fellowship Grant, Ref C19221/A6086. 
G.A.B. is funded by a Cancer Research UK Programme Grant, Ref C237/A6295. Cancer Research UK is a charity registered in the United Kingdom, No. 1089464. D.Mc.G. and J.H.N are supported by AstraZeneca. C.J.R. is supported by GlaxoSmithKline.

\section{REFERENCES}

1. Young IR, Clarke GJ, Bailes DR, Pennock JM, Doyle FH, Bydder GM. Enhancement of relaxation rate with paramagnetic contrast agents in NMR imaging. J Comput Tomogr 1981;5:543-547.

2. Ganong W. Review of medical physiology. New York: Lange Medical; 2003 p 669-671.

3. Roughton FJ, Severinghaus JW. Accurate determination of O2 dissociation curve of human blood above 98.7 percent saturation with data on O2 solubility in unmodified human blood from 0 degrees to 37 degrees C. J Appl Physiol 1973;35:861-869.

4. West JB. Respiratory physiology: the essentials. Philadelphia: Lippincott Williams \& Wilkins; 2005 p. 75-92.

5. Edelman RR, Hatabu H, Tadamura E, Li W, Prasad PV. Noninvasive assessment of regional ventilation in the human lung using oxygenenhanced magnetic resonance imaging. Nat Med 1996;2:1236-1239.

6. Hatabu H, Tadamura E, Chen Q, Stock KW, Li W, Prasad PV, Edelman RR. Pulmonary ventilation: dynamic MRI with inhalation of molecular oxygen. Eur J Radiol 2001;37:172-178.

7. Naish JH, Parker GJ, Beatty PC, Jackson A, Young SS, Waterton JC, Taylor CJ. Improved quantitative dynamic regional oxygen-enhanced pulmonary imaging using image registration. Magn Reson Med 2005; 54:464-469.

8. Ohno Y, Koyama H, Nogami M, Obara M, Kawamitsu M, Takenaka D, Cauteren MV, Hatabu H, Sugimura K. Wash-in time of molecular oxygen from dynamic oxygen-enhanced MRI: new approach for assessment of smoking-related pulmonary functional loss. In: Proceedings of the 14th Annual Meeting of ISMRM, Seattle, WA, USA, 2006 (Abstract 33).

9. Janne d'Othee B, Rachmuth G, Munasinghe J, Lang EV. The effect of hyperoxygenation on T1 relaxation time in vitro. Acad Radiol 2003;10: $854-860$.

10. Tadamura E, Hatabu H, Li W, Prasad PV, Edelman RR. Effect of oxygen inhalation on relaxation times in various tissues. J Magn Reson Imaging 1997;7:220-225

11. Jones RA, Ries M, Moonen CT, Grenier N. Imaging the changes in renal T1 induced by the inhalation of pure oxygen: a feasibility study. Magn Reson Med 2002;47:728-735.

12. Matsumoto K, Bernardo M, Subramanian S, Choyke P, Mitchell JB, Krishna MC, Lizak MJ. MR assessment of changes of tumor in response to hyperbaric oxygen treatment. Magn Reson Med 2006;56:240-246.

13. Duling BR. Microvascular responses to alterations in oxygen tension. Circ Res 1972;31:481-489.

14. Rousseau A, Bak Z, Janerot-Sjoberg B, Sjoberg F. Acute hyperoxaemiainduced effects on regional blood flow, oxygen consumption and central circulation in man. Acta Physiol Scand 2005;183:231-240.

15. Kruuv JA, Inch WR, McCredie JA. Blood flow and oxygenation of tumors in mice. I. Effects of breathing gases containing carbon dioxide at atmospheric pressure. Cancer 1967;20:51-59.

16. Haase A. Snapshot FLASH MRI. Applications to T1, T2, and chemicalshift imaging. Magn Reson Med 1990;13:77-89.

17. Zhu XP, Li KL, Kamaly-Asl ID, Checkley DR, Tessier JJ, Waterton JC, Jackson A. Quantification of endothelial permeability, leakage space, and blood volume in brain tumors using combined T1 and T2* contrastenhanced dynamic MR imaging. J Magn Reson Imaging 2000;11:575-585.

18. Bluml S, Schad LR, Stepanow B, Lorenz WJ. Spin-lattice relaxation time measurement by means of a TurboFLASH technique. Magn Reson Med 1993;30:289-295.

19. de Certaines JD, Henriksen O, Spisni A, Cortsen M, Ring PB. In vivo measurements of proton relaxation times in human brain, liver, and skeletal muscle: a multicenter MRI study. Magn Reson Imaging 1993 11:841-850.

20. Noseworthy MD, Kim JK, Stainsby JA, Stanisz GJ, Wright GA. Tracking oxygen effects on MR signal in blood and skeletal muscle during hyperoxia exposure. J Magn Reson Imaging 1999;9:814-820.

21. Schenk WG, Jr, Mc DJ, Mc DK, Drapanas T. Direct measurement of hepatic blood flow in surgical patients: with related observations on hepatic flow dynamics in experimental animals. Ann Surg 1962;156:463-471.
22. McGrath DM, Naish JH, Beatty PC, Jackson A, Waterton JC, Taylor CJ, Parker GJM. Measured decrease in T1 relaxation time in skeletal muscle on oxygen inhalation. In: Proceedings of the 14th Annual Meeting of ISMRM, Seattle, WA, USA, 2006 (Abstract 255).

23. Duling BR, Berne RM. Longitudinal gradients in periarteriolar oxygen tension. A possible mechanism for the participation of oxygen in local regulation of blood flow. Circ Res 1970;27:669-678.

24. Tsai AG, Cabrales P, Hangai-Hoger N, Intaglietta M. Oxygen distribution and respiration by the microcirculation. Antioxid Redox Signal 2004;6:1011-1018.

25. de Bazelaire CM, Duhamel GD, Rofsky NM, Alsop DC. MR imaging relaxation times of abdominal and pelvic tissues measured in vivo at 3.0 T: preliminary results. Radiology 2004;230:652-659.

26. Sharkey RA, Mulloy EM, O’Neill SJ. Acute effects of hypoxaemia, hyperoxaemia and hypercapnia on renal blood flow in normal and renal transplant subjects. Eur Respir J 1998;12:653-657.

27. Raitakari M, Knuuti MJ, Ruotsalainen U, Laine H, Makea P, Teras M, Sipila H, Niskanen T, Raitakari OT, Iida H, et al. Insulin increases blood volume in human skeletal muscle: studies using [15O]CO and positron emission tomography. Am J Physiol 1995;269(Pt 1):E1000-E1005.

28. Crawford P, Good PA, Gutierrez E, Feinberg JH, Boehmer JP, Silber DH, Sinoway LI. Effects of supplemental oxygen on forearm vasodilation in humans. J Appl Physiol 1997;82:1601-1606.

29. Kaanders JH, Pop LA, Marres HA, Liefers J, van den Hoogen FJ, van Daal WA, van der Kogel AJ. Accelerated radiotherapy with carbogen and nicotinamide (ARCON) for laryngeal cancer. Radiother Oncol 1998;48:115-122.

30. Griffiths JR, Taylor NJ, Howe FA, Saunders MI, Robinson SP, Hoskin PJ, Powell ME, Thoumine M, Caine LA, Baddeley H. The response of human tumors to carbogen breathing, monitored by gradient-recalled echo magnetic resonance imaging. Int J Radiat Oncol Biol Phys 1997;39:697-701.

31. Taylor NJ, Baddeley H, Goodchild KA, Powell ME, Thoumine M, Culver LA, Stirling JJ, Saunders MI, Hoskin PJ, Phillips H, Padhani AR, Griffiths JR. BOLD MRI of human tumor oxygenation during carbogen breathing. J Magn Reson Imaging 2001;14:156-163.

32. Rijpkema M, Kaanders JH, Joosten FB, van der Kogel AJ, Heerschap A. Effects of breathing a hyperoxic hypercapnic gas mixture on blood oxygenation and vascularity of head-and-neck tumors as measured by magnetic resonance imaging. Int J Radiat Oncol Biol Phys 2002;53: 1185-1191.

33. Hill SA, Collingridge DR, Vojnovic B, Chaplin DJ. Tumour radiosensitization by high-oxygen-content gases: influence of the carbon dioxide content of the inspired gas on PO2, microcirculatory function and radiosensitivity. Int J Radiat Oncol Biol Phys 1998;40:943-951.

34. Hoskin PJ, Abdelath O, Phillips H, Gilligan S, Saunders MI, Broderick $\mathrm{P}$, Baddeley H. Inspired and expired gas concentrations in man during carbogen breathing. Radiother Oncol 1999;51:175-177.

35. Hampson NB, Jobsis-VanderVliet FF, Piantadosi CA. Skeletal muscle oxygen availability during respiratory acid-base disturbances in cats. Respir Physiol 1987;70:143-158.

36. Dewhirst MW, Ong ET, Rosner GL, Rehmus SW, Shan S, Braun RD, Brizel DM, Secomb TW. Arteriolar oxygenation in tumour and subcutaneous arterioles: effects of inspired air oxygen content. Br J Cancer 1996;27:S241-246.

37. Anand IS, Chandrashekhar Y, Ferrari R, Sarma R, Guleria R, Jindal SK, Wahi PL, Poole-Wilson PA, Harris P. Pathogenesis of congestive state in chronic obstructive pulmonary disease. Studies of body water and sodium, renal function, hemodynamics, and plasma hormones during edema and after recovery. Circulation 1992;86:12-21.

38. Irie H, Tatsumi T, Takamiya M, Zen K, Takahashi T, Azuma A, Tateishi K, Nomura T, Hayashi H, Nakajima N, Okigaki M, Matsubara H. Carbon dioxide-rich water bathing enhances collateral blood flow in ischemic hindlimb via mobilization of endothelial progenitor cells and activation of NO-cGMP system. Circulation 2005;111:1523-1529.

39. Falk SJ, Ward R, Bleehen NM. The influence of carbogen breathing on tumour tissue oxygenation in man evaluated by computerised p02 histography. Br J Cancer 1992;66:919-924.

40. Diergarten T, Martirosian P, Kottke R, Vogel U, Stenzl A, Claussen CD Schlemmer HP. Functional characterization of prostate cancer by integrated magnetic resonance imaging and oxygenation changes during carbogen breathing. Invest Radiol 2005;40:102-109.

41. Neeman M, Dafni H, Bukhari O, Braun RD, Dewhirst MW. In vivo BOLD contrast MRI mapping of subcutaneous vascular function and maturation: validation by intravital microscopy. Magn Reson Med 2001;45:887-898. 